\title{
ON A CLASS OF HYPERFUCHSIAN FUNCTIONS*
}

\author{
BY \\ CLYDE FIRMAN CRAIG
}

\section{Introduction.}

As a generalization of the fuchsian functions, PiCard introduced the hyperfuchsian. These he defined $\dagger$ as uniform functions of two independent complex variables which are absolutely invariant for an infinite discontinuous group of linear fractional transformations on the variables. Moreover the group, when expressed in its homogeneous form, is such as to leave an indefinite Hermitian form invariant.

In a series of memoirs $\ddagger$ PICARD has established the existence and general theory of such functions. In a particular example he uses as variables the ratios of the values of the integral

$$
\int[z(z-1)(z-x)(z-y)]^{-\frac{1}{3}} d z
$$

taken along three independent contours in the associated Riemann surface, and generates the group by the motions of the two branch-points $x$ and $y$. Following and extending PICARv's study of this particular group, Alezais $\S$ has considered the Riemann surface

$$
w^{3}=(z-\alpha)(z-\beta)(z-\gamma)(z-\delta)
$$

and the values of the moduli of periodicity of a set of associated linearly independent integrals of the first kind along a properly chosen system of cross-cuts on the surface. The group is generated by the monodromy of the four branchpoints $\alpha, \beta, \gamma, \delta$, and contains PiCard's group as a subgroup. Considering the Picard group to be of special interest, Alezais studies it in some detail and

* Presented to the Society, December 31, 1908.

†A cta Mathematica, vol. 5 (1885), p. 121. See also R. AlezaIs, Sur une classe de fonctions hyperfuchsiennes, etc., Paris (1901), p. 1. This thesis contains (p. 3) a complete bibliography of Picard's papers on the problem. An abridgment of the thesis under the same title is in the Annales de l'École Normale Supérieure, (3) vol. 19 (1902), p. 261.

$\ddagger$ Aota Mathematica, vol. 2 (1882), p. 1, and vol. 5 (1885), p. 21; also several notes in Comptes Rendus, 1882-1885.

§ Loc. cit. 
constructs quotients of the products of theta-functions of half-integer characteristics and zero arguments as hyperfuchsian functions belonging to the group.

The only other binomial Riemann surface of genus 3 having five branch-points may be written

$$
w^{4}=(z-a)(z-b)(z-c)^{2}(z-d)^{2}(z-e)^{2} .
$$

The present paper determines the monodromy group of this surface and a set of hyperfuchsian functions belonging to its group. The fourth section contains an extension of a known result on the number of linearly independent moduli of an abelian integral of the first kind connected with a binomial Riemann surface. In the final section an upper limit is fixed for the number of generators of monodromy groups connected with binomial Riemann surfaces, and a method is given for picking out a set of transformations from which the generators may be determined.

\section{§1. The monodromy group.}

Consider the Riemann surface of genus 3,

$$
w^{4}=(z-a)(z-b)(z-c)^{2}(z-d)^{2}(z-e)^{2},
$$

where $a, b, c, d, e$ may be assumed finite. We will denote the different values of $w$ corresponding to the same value of $z$ by $w_{1}, w_{2}, w_{3}, w_{4}$, and will assign the branch $w_{m}$ to sheet $m$ of the surface. The several values of $w$ will be connected by the relation $w_{m}=i^{m-1} w_{1}$.

A set of linearly independent integrals of the first kind is selected as follows:

$$
\begin{gathered}
u_{1}=\int \frac{d z}{w} ; \quad u_{2}=\int(z-c)(z-d)(z-e) \frac{d z}{w^{3}} ; \\
u_{3}=\int z(z-c)(z-d)(z-e) \frac{d z}{w^{3}} .
\end{gathered}
$$

The branch-lines and cross-cuts for the surface are chosen as indicated in Fig. 1.
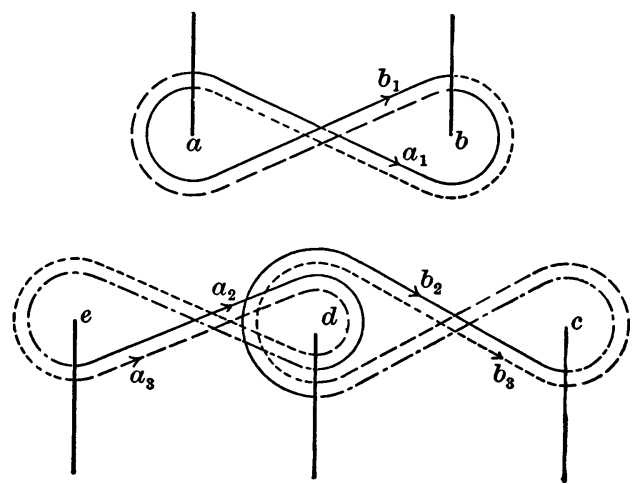

FIG. 1.
Sheet 1

Sheet 2

Sheet 3

Sheet 4 
Denoting the results of integrating $u_{i}$ along the paths $a_{j}, b_{j}$ by $A_{j}^{(i)}, B_{j}^{(i)}$ respectively, the table of moduli of periodicity of the integrals (2) is

\begin{tabular}{c|ccc|ccc} 
& $a_{1}$ & $a_{2}$ & $a_{3}$ & $b_{1}$ & $b_{2}$ & $b_{3}$ \\
\hline$u_{1}$ & $i A_{1}^{(1)}$ & $-B_{2}^{(1)}$ & $i B_{2}^{(1)}$ & $A_{1}^{(1)}$ & $A_{2}^{(1)}$ & $-i A_{2}^{(1)}$ \\
$u_{2}$ & $-i A_{1}^{(2)}$ & $-B_{2}^{(2)}$ & $-i B_{2}^{(2)}$ & $A_{1}^{(2)}$ & $A_{2}^{(2)}$ & $i A_{2}^{(2)}$ \\
$u_{3}$ & $-i A_{1}^{(3)}$ & $-B_{2}^{(3)}$ & $-i B_{2}^{(3)}$ & $A_{1}^{(3)}$ & $A_{2}^{(3)}$ & $i A_{2}^{(3)}$
\end{tabular}

subject to the conditions

$$
\begin{aligned}
& i A_{1}^{(1)} A_{1}^{(2)}-A_{2}^{(2)} B_{2}^{(1)}+A_{2}^{(1)} B_{1}^{(2)}=0, \\
& i A_{1}^{(1)} A_{1}^{(3)}-A_{2}^{(3)} B_{2}^{(1)}+A_{2}^{(1)} B_{1}^{(3)}=0 .
\end{aligned}
$$

The normal table of moduli assumes the form

\begin{tabular}{c|ccc|ccc} 
& $a_{1}$ & $a_{2}$ & $a_{3}$ & $b_{1}$ & $b_{2}$ & $b_{3}$ \\
\hline$v_{1}$ & $\pi i$ & 0 & 0 & $\pi i(i)$ & $\pi i(-u)$ & $\pi i(-i u)$ \\
$v_{2}$ & 0 & $\pi i$ & 0 & $\pi i(-u)$ & $\pi i\left(\frac{-i u^{2}}{2}-v\right)$ & $\pi i\left(\frac{u^{2}}{2}\right)$ \\
$v_{3}$ & 0 & 0 & $\pi i$ & $\pi i(-i u)$ & $\pi i\left(\frac{u^{2}}{2}\right)$ & $\pi i\left(\frac{i u^{2}}{2}-v\right)$
\end{tabular}

where

$$
u=\frac{A_{1}^{(1)}}{B_{2}^{(1)}}, \quad v=\frac{A_{2}^{(1)}}{B_{2}^{(1)}}
$$

Denote by $a_{i k}^{\prime}$ the real part of the element of this table which represents the modulus of periodicity of the integral $v_{k}$ at the cut $b_{i}$. If $r_{i}, r_{k}$ are integers, then $* \sum_{i k} a_{i k}^{\prime} r_{i} r_{k}$ must be negative; this condition will be satisfied if $\dagger$

$$
u \bar{u}-i(v-\bar{v})<0,
$$

wherein $\bar{u}, \bar{v}$, are the conjugates of $u, v$.

We now define the group $G$ which we desire to investigate as the totality of linear fractional transformations on the variables $u, v$ due to the monodromy of the branch-points of the given surface (1). The branch-points are supposed to move continuously over the plane in such a way that at the end of the movement they occupy as a whole their original positions and the Riemann surface has returned to its original form. Since $u=A_{1}^{(1)} / B_{2}^{(1)}, v=A_{2}^{(1)} / B_{2}^{(1)}$, and $A_{1}^{(1)}, A_{2}^{(1)}, B_{2}^{(1)}$ are the values of the integral $u_{1}$ along the paths $a_{1}, a_{2}, b_{2}$ respectively, in determining the transformation on $u, v$ due to any movement of the

* STAHL, Abelsche Funktionen, p. 122 (XV).

$\dagger$ KRAZER, Thetafunktionen, p. 17 (XVII). 
branch-points, it will be necessary merely to investigate the value of $u_{1}$ along the paths $a_{1}^{\prime}, a_{2}^{\prime}, b_{2}^{\prime}$, which will denote the paths into which $a_{1}, a_{2}, b_{2}$ have been changed. For brevity write

$$
x=A_{1}^{(1)}, \quad y=A_{2}^{(1)}, \quad z=B_{2}^{(1)} .
$$

The values of $u_{1}$ along the new paths will be denoted by $x^{\prime}, y^{\prime}, z^{\prime}$ respectively, and the variables $u, v$ will have been transformed into the variables $u^{\prime}, v^{\prime}$, where

$$
u^{\prime}=\frac{x^{\prime}}{z^{\prime}}, \quad v^{\prime}=\frac{y^{\prime}}{z^{\prime}} .
$$

By a positive interchange of two branch-points we shall mean that the positions are interchanged in such a manner that the line joining the two shall have been rotated counter-clockwise about its middle point. When necessary the actual paths of the points interchanged are supposed to vary from the circle described by the end points of the joining line sufficiently to prevent any third point being enclosed in the completed path of the two points. A point will be said to move around another point positively when the enclosed area is to the left of the moving point; this area will be assumed to enclose but a single branchpoint unless the contrary is stated. Denote by $(\kappa \lambda)$ the transformation on the variables arising from a positive interchange of the two points $\kappa$ and $\lambda$. This same notation will be used to denote the motion of $\kappa$ positively around $\lambda$ if $\kappa$ and $\lambda$ cannot be interchanged under the above restrictions on the movement of the branch-points.

By interchanging the branch-points $a$ and $b$ positively, it is readily found that $x, y, z$ undergo the transformation

whence

$$
x^{\prime}=i x, \quad y^{\prime}=y, \quad z^{\prime}=z ;
$$

$$
u^{\prime}=-\frac{u}{i}, \quad v^{\prime}=\frac{i v}{i},
$$

the coefficients being so written that their determinant is unity. Similarly we obtain

$$
\begin{gathered}
u^{\prime}=u, \quad v^{\prime}=v-1, \\
u^{\prime}=\frac{u}{v+1}, \quad v^{\prime}=\frac{v}{v+1}, \\
u^{\prime}=\frac{-u+1+i}{i}, \quad v^{\prime}=\frac{(-1+i) u+i v+1-i}{i} .
\end{gathered}
$$

Every other monodromy of the branch-points which leaves the surface unaltered may be considered as a combination of the above four taken in proper 
order, and consequently the transformation arising from such a monodromy may be expressed in terms of the above four. To show this we may proceed as follows, distinguishing two cases, namely, when a single pair of branch-points is moved, and when more than two are moved.

Under the first case we have operations such as interchanging $c$ and $e$, or moving $b$ around $d$. To produce the motion of $b$ around $d$, for instance, we may perform in succession $(a b)^{-1},(d e),(a e),(d e)^{-1},(a b)$; or $(d e),(a b)^{-1}$, $(a e),(a b),(d e)^{-1}$. Thus for our transformation on the variables we have

$$
(b d)=(a b)^{-1}(d e)(a e)(d e)^{-1}(a b)=(d e)(a b)^{-1}(a e)(a b)(d e)^{-1},
$$

where the transformations on the variables are applied from right to left as written. The last written equality may be easily verified; for $(a b)$ and (de) are commutative, as are their powers, since the corresponding motions on the surface may be performed simultaneously without interference. Suppose now that one of the branch-points, say $a$, performs any circuit whatsoever and either returns to its original position or makes the possible interchange with the branch-point $b$. We may resolve its path (by proper deformation) into a series of circuits around the remaining branch-points singly, together with the possible interchange at the end. Since each of these circuits about the single branchpoints and the interchange are severally expressible in terms of the four fundamental motions, it follows that the transformation due to the motion of $a$ is expressible in terms of the four fundamental transformations. So with each of the other points $b, c, d, e$.

In case two or more branch-points move at the same time, no difficulty will arise if the paths do not intersect. For the paths are traversed independently and the movement giving rise to one path may be considered as having been completed before any other branch-point begins to move. The above argument shows that the transformation arising from these several motions can be expressed in terms of the four fundamental ones. If the paths of the moving points do intersect, we must specify the order in which two or more branch-points pass through a given point of intersection. For two branch-points must not coincide, and moreover the order specified must be compatible with the geometric possibilities of the surface. When this order is established the portions of the paths between the points of intersection may be considered as new paths of the form treated above. At the points of intersection we have simply an interchange of two points or one point moving around another. Since any such motion can now be resolved into the four fundamental ones, each taken the proper number of times and in the proper order, it follows that the transformation arising from any monodromy of the branch-points can be expressed in terms of $(a b),(c d)$, $(d e),(a e)$. 
These four fundamental transformations are expressible in terms of the two,

$$
A \equiv\left|\begin{array}{ccc}
1 & 1-i & 0 \\
1-i & -1-i & -1 \\
0 & 1 & 0
\end{array}\right|, \quad B \equiv\left|\begin{array}{ccc}
1 & 0 & 0 \\
0 & 1 & -1 \\
0 & 0 & 1
\end{array}\right|,
$$

the determinant of the coefficients only being written. The expressions are

$$
\begin{aligned}
& (a b)=B A^{5} B^{-1} A^{5} B A, \\
& (c d)=B, \\
& (d e)=A^{-1} B A, \\
& (a e)=A^{-2} B^{-1} A^{-2} B^{-1} .
\end{aligned}
$$

Moreover $A$ is a transformation of the given group, since

$$
A=(a e)(c d)(d e)(a b)^{-1}(c d) .
$$

Since $B$ is non-periodic, the theorem follows:

The monodromy group $G$ connected with the Riemann surface (1) is of infinite order and is generated by the two operators, $A$ and $B$, defined by (6). The quadratic form $u \bar{u}-i(v-\bar{v})$ is a relative invariant for the group $G$.

\section{§2. The arithmetic form of the group.}

Since the coefficients of the generating transformations of $G$ are complex integers, that is, are of the form $m+n i$, where $m$ and $n$ are integers, it follows that every transformation of $G$ has complex integers for coefficients. We wish to characterize further these coefficients.

For this purpose write the general transformation of $G$ in the form

$$
\text { (7) } u^{\prime}=\frac{a_{1} u+b_{1} v+c_{1}}{\Delta}, \quad v^{\prime}=\frac{a_{2} u+b_{2} v+c_{2}}{\Delta}, \quad \Delta=a_{3} u+b_{3} v+c_{3} \text {, }
$$

where the coefficients are complex integers with determinant unity. Since the monodromy group leaves $\phi=i(v-\bar{v})-u \bar{u}$ relatively invariant, the transformed form $\phi^{\prime}$ will be connected with the original by the relation

$$
\phi^{\prime}=\frac{\phi}{\Delta \bar{\Delta}},
$$

where $\bar{\Delta}$ is the conjugate of $\Delta$. In order that this relation may subsist the coefficients in (7) must satisfy the conditions

$$
\begin{array}{ll}
a_{1} \bar{a}_{1}-i\left(a_{2} \bar{a}_{3}-\bar{a}_{2} a_{3}\right)=1, & a_{1} \bar{b}_{1}-i\left(a_{2} \bar{b}_{3}-a_{3} \bar{b}_{2}\right)=0, \\
b_{1} \bar{b}_{1}-i\left(b_{2} \bar{b}_{3}-\bar{b}_{2} b_{3}\right)=0, & a_{1} \bar{c}_{1}-i\left(a_{2} \bar{c}_{3}-a_{3} \bar{c}_{2}\right)=0, \\
c_{1} \bar{c}_{1}-i\left(c_{2} \bar{c}_{3}-\bar{c}_{3} c_{2}\right)=0, & b_{1} \bar{c}_{1}-i\left(b_{2} \bar{c}_{3}-b_{3} \bar{c}_{2}\right)=-i .
\end{array}
$$


Using the relations (8) to solve (7), we obtain

$$
\begin{aligned}
& \frac{u}{\Delta}=\bar{a}_{1} u^{\prime}-i \bar{a}_{3} v^{\prime}+i \bar{a}_{2}, \\
& \frac{v}{\Delta}=i \bar{c}_{1} u^{\prime}+\bar{c}_{3} v^{\prime}-\bar{c}_{2}, \\
& \frac{1}{\Delta}=-i \bar{b}_{1} u^{\prime}-\bar{b}_{3} u+\bar{b}_{2}=\Delta^{\prime},
\end{aligned}
$$

which give the inverse of transformation (7). On account of the relative invariance of $\phi^{\prime}$, the coefficients of $\left(7^{\prime}\right)$ must satisfy the relations

$$
\begin{array}{ll}
a_{1} \bar{a}_{1}+i\left(b_{1} \bar{c}_{1}-\bar{b}_{1} c_{1}\right)=1, & a_{1} \bar{a}_{2}+i\left(b_{1} \bar{c}_{2}-\bar{b}_{2} c_{1}\right)=0, \\
a_{2} \bar{a}_{2}+i\left(b_{2} \bar{c}_{2}-\bar{b}_{2} c_{2}\right)=0, & a_{1} \bar{a}_{3}+i\left(b_{1} \bar{c}_{3}-\bar{b}_{3} c_{1}\right)=0, \\
a_{3} \bar{a}_{3}+i\left(b_{3} \bar{c}_{3}-\bar{b}_{3} c_{3}\right)=0, & a_{2} \bar{a}_{3}+i\left(b_{2} \bar{c}_{3}-\bar{b}_{3} c_{2}\right)=i .
\end{array}
$$

Denote by $A_{1}, \ldots$ the minors of $a_{1}, \ldots$ in the determinant of $(7)$; solve (7) for $u / \Delta, v / \Delta, 1 / \Delta$, and we obtain

$$
\begin{aligned}
& \frac{u}{\Delta}=A_{1} u^{\prime}+A_{2} v^{\prime}+A_{3}, \\
& \frac{v}{\Delta}=B_{1} u^{\prime}+B_{2} v^{\prime}+B_{3}, \\
& \frac{1}{\Delta}=C_{1} u^{\prime}+C_{2} v^{\prime}+C_{3} .
\end{aligned}
$$

Comparing this form of the inverse transformation with $\left(7^{\prime}\right)$, we obtain the relations

$$
\begin{array}{cl}
\bar{a}_{1}=b_{2} c_{3}-b_{3} c_{2}=A_{1}, & \bar{b}_{1}=i\left(a_{2} b_{3}-a_{3} b_{2}\right)=i C_{1}, \\
\bar{a}_{2}=-i\left(b_{1} c_{2}-b_{2} c_{1}\right)=-i A_{3}, & \bar{b}_{2}=a_{1} b_{2}-a_{2} b_{1}=C_{3}, \\
\bar{a}_{3}=-i\left(b_{1} c_{3}-b_{3} c_{1}\right)=i A_{2}, & \bar{b}_{3}=a_{1} b_{3}-a_{3} b_{1}=-C_{2}, \\
\bar{c}_{1}=i\left(a_{2} c_{3}-a_{3} c_{2}\right)=-i B_{1}, \\
\bar{c}_{2}=a_{1} c_{2}-a_{2} c_{1}=-B_{3}, \\
\bar{c}_{3}=a_{1} c_{3}-a_{3} c_{1}=B_{2} .
\end{array}
$$

These relations may be solved for $a_{1}, a_{2}, a_{3}$ (or $a_{1}, b_{1}, c_{1}$ ) in terms of the other elements, which will be connected by three bilinear relations taken from ( $\left.8^{\prime}\right)$ [or (8)]. 
Since $\left(a_{2} \bar{a}_{3}-\bar{a}_{2} a_{3}\right)$ and similar expressions are divisible by 2 , from (8) and $\left(8^{\prime}\right)$ it follows that

$a_{1} \bar{a}_{1} \equiv 1, \quad a_{2} \bar{a}_{2} \equiv 0, \quad a_{3} \bar{a}_{3} \equiv 0, \quad b_{1} \bar{b}_{1} \equiv 0, \quad c_{1} \bar{c}_{1} \equiv 0 \quad(\bmod 2)$, from which

$$
\begin{array}{rll}
a_{1}=2 m+1+2 n i & \text { or } & 2 m+(2 n+1) i, \\
a_{2} \equiv 0, \quad a_{3} \equiv 0, \quad b_{\mathrm{i}} \equiv 0, & c_{1} \equiv 0 \quad[\bmod (1+i)] .
\end{array}
$$

From the relation $\bar{b}_{2}=a_{1} b_{2}-a_{2} b_{1}$ it follows that

$$
\bar{b}_{2}-a_{1} b_{2} \equiv 0 \quad(\bmod 2) \text {. }
$$

If $a_{1}=2 m+1+2 n i$, this congruence gives $\bar{b}_{2}-b_{2} \equiv 0(\bmod 2)$. If $a_{1}=2 m+(2 n+1) i$, the above congruence becomes

$$
\bar{b}_{2}-i b_{2} \equiv 0 \quad(\bmod 2) \text {. }
$$

If we write $b_{2}=b_{2}^{\prime}+i b_{2}^{\prime \prime}$, this congruence becomes

$$
(1-i)\left(b_{2}^{\prime}+b_{2}^{\prime \prime}\right) \equiv 0 \quad(\bmod 2),
$$

whence $b_{2}^{\prime}$ and $b_{2}^{\prime \prime}$ are of the same parity, and therefore $b_{2} \equiv 0[\bmod (1+i)]$. A similar argument applied to the other relations (9) yields the conditions

$$
b_{3} \equiv 0, \quad c_{2} \equiv 0, \quad c_{3} \equiv 0 \quad[\bmod (1+i)] .
$$

Consequently $b_{2} c_{3}-b_{3} c_{2} \equiv 0(\bmod 2)$. But $\bar{a}_{1}=b_{2} c_{3}-b_{3} c_{2} \equiv 0(\bmod 2)$, which violates the assumption

$$
a_{1}=2 m+(2 n+1) i,
$$

and hence this value of $a_{1}$ is impossible. We accordingly have

$$
a_{1} \equiv 1 \quad(\bmod 2) .
$$

Therefore $a_{1}=1+2 m+2 n i$, and by (8) and (9),

$$
b_{2} c_{3}-b_{3} c_{2} \equiv 1, \quad b_{2} \bar{c}_{3}-\bar{b}_{3} c_{2} \equiv 1 \quad(\bmod 2) \text {. }
$$

The residues of complex integers $(\bmod 2)$ are $0,1, i, 1+i$. The two above congruences reduce the possible combinations of residues of $b_{2}, b_{3}, c_{2}, c_{3}$ to 48 . Using these 48 possible residues in connection with the equations (9), we are led to 192 transformations which are incongruent modulo 2 .

By using the relations (9) the periods of the transformations of $G$ may be readily determined. The characteristic equation for the general transformation of the group is

$$
\left|\begin{array}{ccc}
a_{1}-\rho & b_{1} & c_{1} \\
a_{2} & b_{2}-\rho & c_{2} \\
a_{3} & b_{3} & c_{3}-\rho
\end{array}\right|=0
$$


or, by (9),

$$
\rho^{3}-d \rho^{2}+d \rho-1=0, \quad d=a_{1}+b_{2}+c_{3}=a+b i,
$$

$a$ and $b$ being integers. If the transformation is periodic, all the roots of the characteristic equation must be roots of unity, and conversely. Hence $|d| \leqq 3$. Let $\rho=(1+i t) /(1-i t)$, and the characteristic equation becomes

$$
(1+a) t^{3}+b t^{2}+(a-3) t+b=0 .
$$

If the roots of this equation are real, those of the characteristic equation are roots of unity, and conversely. The roots of this equation will be real if

where

$$
G^{2}+4 H^{3} \leqq 0
$$

$$
\begin{aligned}
& G=\frac{2}{27}\left(9 a^{2} b+36 a b+27 b+b^{3}\right), \\
& H=\frac{3(a+1)(a-3)-b^{2}}{9} .
\end{aligned}
$$

The possible values of $d$ for which this equation is satisfied are $-1,0,1$, $-1 \pm 2 i, 2, \pm i,-1 \pm i, 3$. Construct the corresponding equation in $\rho$ and factor ; the periods follow immediately. With the notation

$$
\varepsilon=e^{\frac{\pi i}{3}}, \quad i=e^{\frac{\pi i}{2}}, \quad j=e^{\frac{\pi i}{4}}
$$

the tabulated results are:

\begin{tabular}{c|c|c|c|c|c|c|c|c}
\hline$d$ & -1 & 0 & 1 & $-1 \pm 2 i$ & 2 & $\pm i$ & $-1 \pm i$ & 3 \\
\hline$\rho$ & $1,-1,-1$ & $1,-\varepsilon, \varepsilon^{2}$ & $1, i,-i$ & $\begin{array}{c}-1, \pm i, \\
\pm i\end{array}$ & $1, \varepsilon,-\varepsilon^{2}$ & $i, j,-j$, & $-1, \pm i \varepsilon$, & $1,1,1$ \\
Period & $2, \infty$ & 3 & 4 & $4, \infty$ & 6 & 8 & 12 & $\infty$ \\
\hline
\end{tabular}

For $d=-1,-1 \pm 2 i$ all second order minors of the determinant of the characteristic equation must vanish for the double root if the period is finite. For all values of $d$ other than the above the substitution is non-periodic.*

* The group $G$, considered as abstract, is apparently characterized by only the conditions of this section. The problom arises : Is $G$ the maximum group which satisfies these conditions? The question is as yet unanswered ; but there is good reason to believe that $G$ is the maximum group. If we transform $G$ into a group $G^{\prime}$ by mesis of

$$
\left|\begin{array}{rrr}
-i & 1 & 1 \\
-1 & 0 & -i \\
0 & 1 & 1
\end{array}\right|
$$

then the coefficients of each linear transformation in $G^{\prime}$ will be complex integers with determinant 1. Moreover the invariant loous for the new group is the hypersphere $u \bar{u}+v \bar{v}=1$. The group $G^{\prime}$ is apparently the maximum group of substitutions having these restrictions, and would acordingly be the simplest analogue, in the case of two variables, to the modular group of linear transformations on a single complex variable. 


\section{§ 3. The hyperfuchsian functions.}

Using the general transformation of the group $G$ in its homogeneous form

$$
\begin{aligned}
& x^{\prime}=a_{1} x+b_{1} y+c_{1} z, \\
& y^{\prime}=a_{2} x+b_{2} y+c_{2} z, \\
& z^{\prime}=a_{3} x+b_{3} y+c_{3} z,
\end{aligned}
$$

we see that corresponding to this linear transformation on the variables, the elements of the table of moduli (3) undergo a linear transformation. The corresponding transformation on the allied theta series requires the determination of a set of integral coefficients $a_{\iota \kappa}, \beta_{\iota \kappa}, \gamma_{\iota k}, \delta_{\iota \kappa}$ which satisfy the identities

$$
\begin{aligned}
-B_{\kappa}^{(i)} & =\sum_{\mu=1}^{3}\left(-a_{\kappa \mu} B_{\mu}^{(i)}+\beta_{\kappa \mu} A_{\mu}^{(i)}\right), \\
A_{\kappa}^{\left({ }^{2}\right)} & =\sum_{=}^{3}\left(-\gamma_{\kappa \mu} B_{\mu}^{(i)}+\delta_{\kappa \mu} A_{\mu}^{(i)}\right),
\end{aligned}
$$

wherein $A_{\kappa}^{(i)}$, etc., have the meanings assigned in $\S 1$, and $A_{\kappa}^{(i)}$ is the value of $A_{\kappa}^{(i)}$ after transformation. Replacing $A_{\kappa}^{(i)}, A_{\kappa}^{(i)}$, etc., in the above identities by their values given by $(5)$ and $\left(7^{\prime \prime}\right)$, the theta transformation is given by

$$
\begin{array}{rrr|rrr}
\alpha_{11} & \alpha_{12} & \alpha_{13} & \beta_{11} & \beta_{12} & \beta_{13} \\
\alpha_{21} & \alpha_{22} & \alpha_{23} & \beta_{21} & \beta_{22} & \beta_{23} \\
-\beta_{21} & -\alpha_{23} & \alpha_{22} & \alpha_{21} & -\beta_{23} & \beta_{22} \\
\hline-\beta_{11} & -\alpha_{13} & \alpha_{12} & \alpha_{11} & -\beta_{13} & \beta_{12} \\
\gamma_{21} & \gamma_{22} & \gamma_{23} & \delta_{21} & \delta_{22} & \delta_{23} \\
-\delta_{21} & -\gamma_{23} & \gamma_{22} & \gamma_{21} & -\delta_{23} & \delta_{22}
\end{array}
$$

wherein the coefficients are subject to certain well-known bilinear relations, and are connected with the coefficients of $\left(7^{\prime \prime}\right)$ by means of the relations

$$
\begin{array}{lll}
a_{1}=\alpha_{11}-\beta i_{11}, & b_{1}=-\beta_{13}-i \beta_{12}, & c_{1}=\alpha_{13}+i \alpha_{12}, \\
a_{2}=\delta_{21}+i \gamma_{21}, & b_{2}=\delta_{22}-i \delta_{23}, & c_{2}=-\gamma_{22}+i \gamma_{23}, \\
a_{3}=-\beta_{21}-i \alpha_{21}, & b_{3}=-\beta_{22}-i \beta_{23}, & c_{3}=\alpha_{22}-i \alpha_{23} .
\end{array}
$$

The bilinear relations mentioned above, when taken by columns, reduce to the following :

$$
\begin{aligned}
\alpha_{11}^{2}+2 \alpha_{21} \delta_{21}+\beta_{11}^{2}-2 \beta_{21} \gamma_{21} & =1, \\
-\alpha_{12} \beta_{13}+\alpha_{13} \beta_{12}+\alpha_{22} \delta_{22}+\alpha_{23} \delta_{23}-\beta_{22} \gamma_{22}-\beta_{23} \gamma_{23} & =1,
\end{aligned}
$$




$$
\begin{aligned}
\alpha_{11} \alpha_{13}-\alpha_{12} \beta_{11}-\alpha_{21} \gamma_{22}+\alpha_{22} \gamma_{21}+\alpha_{23} \delta_{21}-\beta_{21} \gamma_{23} & =0, \\
\alpha_{11} \alpha_{12}+\alpha_{13} \beta_{11}+\alpha_{21} \gamma_{23}+\alpha_{22} \delta_{21}-\alpha_{23} \gamma_{21}-\beta_{21} \gamma_{22} & =0, \\
\alpha_{11} \beta_{13}-\alpha_{21} \delta_{22}-\beta_{11} \beta_{12}-\beta_{21} \delta_{23}+\beta_{22} \gamma_{21}+\beta_{23} \delta_{21} & =0, \\
\alpha_{11} \beta_{12}+\alpha_{21} \delta_{23}+\beta_{11} \beta_{13}-\beta_{21} \delta_{22}+\beta_{22} \delta_{21}-\beta_{23} \gamma_{21} & =0, \\
\alpha_{12} \beta_{12}+\alpha_{13} \beta_{13}+\alpha_{22} \delta_{23}-\alpha_{23} \delta_{22}+\beta_{22} \gamma_{23}-\beta_{23} \gamma_{22} & =0, \\
\alpha_{12}^{2}+\alpha_{13}^{2}+2 \alpha_{22} \gamma_{23}-2 \alpha_{23} \gamma_{22} & =0, \\
\beta_{12}^{2}+\beta_{13}^{2}+2 \beta_{22} \delta_{23}-2 \beta_{23} \delta_{22} & =0 .
\end{aligned}
$$

Rewrite the first, ninth, and eighth of these equations as indicated below. Multiply the sixth equation by $i$, and subtract the fifth from the result. Multiply the fourth by $-i$, and add the third to the result. From the second multiplied by $-i$ subtract the seventh. The results of these operations in the order indicated are

$$
\begin{aligned}
& \left(\alpha_{11}-i \beta_{11}\right)\left(\alpha_{11}+i \beta_{11}\right)-i\left(\delta_{21}+i \gamma_{21}\right)\left(-\beta_{21}+i x_{21}\right)+i\left(\delta_{21}-i \gamma_{21}\right)\left(-\beta_{21}-i \alpha_{21}\right)=1, \\
& \left(-\beta_{13}-i \beta_{12}\right)\left(-\beta_{13}+i \beta_{12}\right)-i\left(\delta_{22}-i \delta_{23}\right)\left(-\beta_{22}-i \beta_{23}\right)+i\left(\delta_{22}+i \delta_{23}\right)\left(-\beta_{22}+i \beta_{23}\right)=0, \\
& \left(\alpha_{13}+i \alpha_{12}\right)\left(\alpha_{13}-i \alpha_{12}\right)-i\left(-\gamma_{22}+i \gamma_{23}\right)\left(\alpha_{22}+i \alpha_{23}\right)+i\left(-\gamma_{22}-i \gamma_{23}\right)\left(\alpha_{22}-i \alpha_{23}\right)=0, \\
& \left(\alpha_{11}-i \beta_{11}\right)\left(-\beta_{13}+i \beta_{12}\right)-i\left(\delta_{21}+i \gamma_{21}\right)\left(-\beta_{22}-i \beta_{23}\right)+i\left(-\beta_{21}-i \alpha_{21}\right)\left(\delta_{22}+i \delta_{23}\right)=0, \\
& \left(\alpha_{11}-i \beta_{11}\right)\left(\alpha_{13}-i \alpha_{12}\right)-i\left(\delta_{21}+i \gamma_{21}\right)\left(\alpha_{22}+i \alpha_{23}\right)+i\left(-\beta_{21}-i \alpha_{21}\right)\left(-\gamma_{22}-i \gamma_{23}\right)=0, \\
& \left(-\beta_{13}-i \beta_{12}\right)\left(\alpha_{13}-i \alpha_{12}\right)-i\left(\delta_{22}-i \delta_{23}\right)\left(\alpha_{22}+i \alpha_{23}\right)+i\left(-\beta_{22}+i \beta_{23}\right)\left(-\gamma_{22}-i \gamma_{23}\right)=-i .
\end{aligned}
$$

In these equations substitute the values of $a_{i}, b_{i}, c_{i}$ from (10), and we obtain the fundamental equations of condition (8). In like manner the bilinear relations obtained by taking the elements of the theta-transformation by rows may be combined to produce a set of equations similar to the above, out of which the conditions of $\left(8^{\prime}\right)$ can be formed.

Since the equations (9) are consequences of the conditions $(8)$ and $\left(8^{\prime}\right)$, we may substitute in (9) the values of $a_{i}, b_{i}, c_{i}$ given by (10). Separating the real and imaginary parts of the resulting equations, we obtain a solution of the bilinear relations connecting the coefficients of the theta transformation. This solution gives six coefficients explicitly in terms of the remaining twelve, which are connected by four bilinear relations.

Because of the particular moduli of the problem at hand certain linear relations exist among the theta functions. To determine what these are we follow the values of any theta function under the transformation which corresponds to. identity in $G$. For this latter $a_{1}=b_{2}=c_{3}, a_{2}=a_{3}=b_{1}=b_{3}=c_{1}=c_{2}=0$. 
Using (10), we find the theta transformation to be

$$
\begin{array}{rrr|rrr}
m & 0 & 0 & n & 0 & 0 \\
0 & m & n & 0 & 0 & 0 \\
0 & -n & m & 0 & 0 & 0 \\
\hline-n & 0 & 0 & m & 0 & 0 \\
0 & 0 & 0 & 0 & m & n \\
0 & 0 & 0 & 0 & -n & m
\end{array}
$$

with $m^{2}+n^{2}=1$, and $m, n$ integers. This transformation yields four cases, viz. : $m=1, n=0 ; m=-1, n=0 ; m=0, n=+1 ; m=0, n=-1$. The corresponding relations between the theta functions with zero arguments are*

$$
\begin{aligned}
& \vartheta\left[\begin{array}{l}
g \\
h
\end{array}\right]((0))=\vartheta\left[\begin{array}{l}
g \\
h
\end{array}\right]((0)), \\
& \vartheta\left[\begin{array}{l}
g \\
h
\end{array}\right]((0))=\vartheta\left[\begin{array}{l}
-g \\
-h
\end{array}\right]((0)), \\
& \vartheta\left[\begin{array}{l}
g \\
h
\end{array}\right]((0))=e^{2 \pi g_{1} h_{1}} \vartheta\left[\begin{array}{rrr}
-h_{1} & g_{3}-g_{2} \\
g_{1} & h_{3}-h_{2}
\end{array}\right]((0)), \\
& \vartheta\left[\begin{array}{l}
g \\
h
\end{array}\right]((0))=e^{2 \pi i g_{1} h_{1}} \vartheta\left[\begin{array}{rr}
h_{1}-g_{3} & g_{2} \\
-g_{1}-h_{3} & h_{2}
\end{array}\right]((0)) .
\end{aligned}
$$

By using the relations (10) the theta-transformations corresponding to the generators of $G$ are determined to be

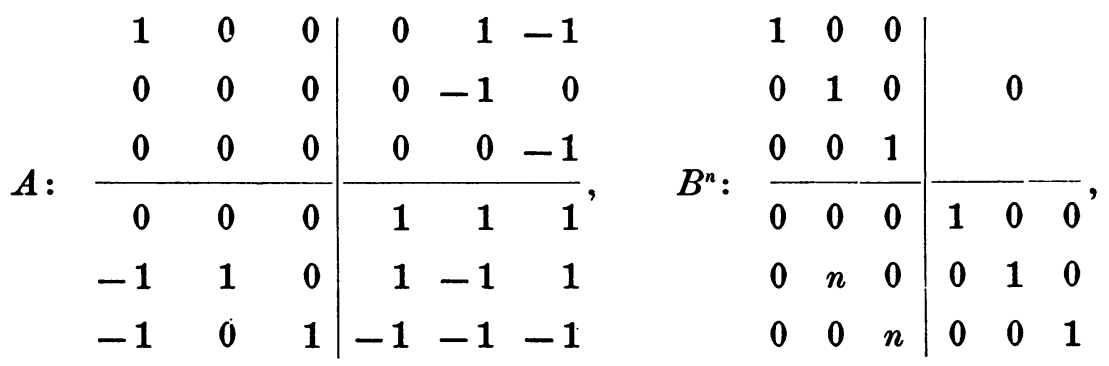

and the corresponding transformations on the theta-functions with zero arguments are $\dagger$ :

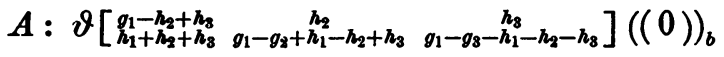

$$
\begin{aligned}
& =i v e^{-2 \pi i\left[h_{2}\left(h_{2}-g_{1}+g_{2}\right)+h_{8}\left(g_{1}-g_{3}\right)\right]} \vartheta\left[\begin{array}{l}
g \\
h
\end{array}\right]((0))_{a},
\end{aligned}
$$

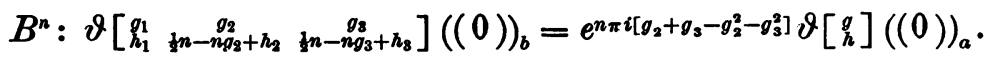

* Krazer u. Prym, Neue Grundlagen einer Theorie der allgemeinen Thetafunktionen, etc., p. 121.

† Loo. cit. 
We now wish to determine the simplest possible products of theta functions with zero arguments which are relatively invariant under the group $G$. If a product of two functions $\vartheta_{1}$ and $\vartheta_{2}$ is relatively invariant, then it follows that $\vartheta_{1}$ is a relative invariant under $A^{2}$ and $B^{2}$. For this to be true, the transformed characteristic must be reducible to one of the four given in (11). In addition to this, the characteristic of $\vartheta_{1}$ must be transformed into that of $\vartheta_{2}$ by $A$ or $B$. This requires further conditions similar to the above. The characteristic of $\vartheta_{1}$ will then be the simultaneous solution of these sets of conditions, if such a solution exists. A similar procedure may be used to determine whether the product of several theta-functions can be relatively invariant under $G$ or not. The following products are believed to be the simplest:

$$
\begin{aligned}
& \phi_{1} \equiv\left[\begin{array}{l}
000 \\
000
\end{array}\right]\left[\begin{array}{l}
000 \\
011
\end{array}\right]\left[\begin{array}{l}
011 \\
000
\end{array}\right], \\
& \phi_{2} \equiv\left[\begin{array}{l}
000 \\
100
\end{array}\right]\left[\begin{array}{l}
000 \\
111
\end{array}\right]\left[\begin{array}{l}
011 \\
100
\end{array}\right]\left[\begin{array}{l}
011 \\
111
\end{array}\right], \\
& \phi_{3} \equiv\left[\begin{array}{l}
000 \\
110
\end{array}\right]\left[\begin{array}{l}
000 \\
101
\end{array}\right]\left[\begin{array}{l}
001 \\
100
\end{array}\right]\left[\begin{array}{l}
001 \\
110
\end{array}\right]\left[\begin{array}{l}
010 \\
100
\end{array}\right]\left[\begin{array}{l}
010 \\
101
\end{array}\right], \\
& \phi_{4} \equiv\left[\begin{array}{l}
000 \\
010
\end{array}\right]\left[\begin{array}{l}
010 \\
000
\end{array}\right]\left[\begin{array}{l}
010 \\
001
\end{array}\right]\left[\begin{array}{l}
101 \\
101
\end{array}\right]\left[\begin{array}{l}
101 \\
111
\end{array}\right]\left[\begin{array}{l}
111 \\
101
\end{array}\right],
\end{aligned}
$$

in which for the sake of brevity we write

$$
\left[\begin{array}{l}
\lambda \\
\mu
\end{array}\right] \equiv \vartheta\left[\begin{array}{c}
\frac{1}{2} \lambda \\
\frac{1}{2} \mu
\end{array}\right]((0))_{a}
$$

( $\lambda, \mu$ integers). The function $\phi \equiv\left[\begin{array}{l}011 \\ 011\end{array}\right]$ is also relatively invariant, but from (11) we find that $\phi=-\phi$, and hence that $\phi \equiv 0$. Denote by $\phi_{i}^{\prime}$ the transformed form of $\phi_{i}$. By using (11) and (12) the following table is deduced for the generating transformations:

\begin{tabular}{c|c|c|c|c}
\hline & $\phi_{1}^{\prime}$ & $\phi_{2}^{\prime}$ & $\phi_{3}^{\prime}$ & $\phi_{4}^{\prime}$ \\
\cline { 2 - 3 } & $-v^{3} \phi_{1}$ & $-v^{4} \phi_{2}$ & $v^{6} \phi_{3}$ & $v^{6} \phi_{4}$ \\
$B$ & $i \phi_{1}$ & $-\phi_{2}$ & $-\phi_{3}$ & $-\phi_{4}$ \\
\hline
\end{tabular}

None of the functions $\phi_{i}$ vanish identically. To show this it will be sufficient to find values of $u, v$ for which some one theta function of each $\phi_{i}$ is different from zero, since the factors of each product are permuted by the transformations of the group. Take the particular values $u=0, v=-i$, which satisfy the condition (4), and substitute in the functions $\left[\begin{array}{l}000 \\ 000\end{array}\right],\left[\begin{array}{l}010 \\ 100\end{array}\right]=\left[\begin{array}{l}100 \\ 000\end{array}\right],\left[\begin{array}{l}010 \\ 100\end{array}\right]=\left[\begin{array}{l}101 \\ 000\end{array}\right]$, 
and $\left[\begin{array}{l}010 \\ 000\end{array}\right]$. This gives

$$
\begin{aligned}
& {\left[\begin{array}{l}
000 \\
000
\end{array}\right]=\sum_{m_{i}=-\infty}^{\infty} e^{-\pi\left[m_{1}^{2}+m_{2}^{2}+m_{3}^{2}\right]},} \\
& {\left[\begin{array}{l}
100 \\
000
\end{array}\right]=\sum_{m_{i}=-\infty}^{\infty} e^{-\pi\left[\left(m_{1}+\frac{1}{2}\right)^{2}+m_{2}^{2}+m_{3}^{2}\right]},} \\
& {\left[\begin{array}{l}
101 \\
000
\end{array}\right]=\sum_{m_{i}=-\infty}^{\infty} e^{-\pi\left[\left(m_{1}+\frac{1}{2}\right)^{2}+m_{2}^{2}+\left(m_{8}+\frac{1}{2}\right)^{2}\right]},} \\
& {\left[\begin{array}{l}
010 \\
000
\end{array}\right]=\sum_{-m_{i}=-\infty}^{\infty} e^{-\pi\left[m_{1}^{2}+\left(m_{2}+\frac{1}{2}\right)^{2}+m_{3}^{2}\right]},}
\end{aligned}
$$

which evidently do not vanish.

None of the functions $\phi_{1}, \phi_{2}, \phi_{3}$ vanishes identically, and the functions

$$
F_{1}=\frac{\phi_{3}}{\phi_{1}^{2}}, \quad F_{2}=\frac{\phi_{4}}{\phi_{1}^{2}}
$$

are absolutely invariant under the group $G$. These functions are believed to be as simple as any of the kind under discussion and belonging to the group.*

\section{§4. Linear independence of the moduli.}

It will be observed that in Table (3) the six moduli of a given integral $u_{i}$ are expressed linearly in terms of three such. This is a particular case of a general theorem which we proceed to deduce.

Denote by

$$
u_{i}=\int \frac{R_{i}(z) d z}{w^{\eta_{i}}}
$$$$
(i=1,2, \cdots, p)
$$

where $R_{i}(z)$ is a rational integral function of $z$, and $\eta_{i}$ is an integer, a system of $p$ linearly independent integrals of the first kind connected with the Riemann surface

$$
w^{\nu}=(z-a)^{a}(z-b)^{\beta} \cdots(z-n)^{\lambda}
$$

having $\mu$ branch-points which may be assumed finite, and with

$$
0<\alpha<\nu, 0<\beta<\nu, \cdots, 0<\lambda<\nu, \alpha+\beta+\cdots+\lambda \equiv 0 \quad(\bmod \nu) .
$$

Denote the different values of $w$ corresponding to the same value of $z$ by $w_{1}, w_{2}, \cdots, w_{\nu}$, and assign the branch $w_{m}$ to the $m$ th sheet of the surface.

* Picard has shown (Acta Mathematica, vol. 5 (1884), p. 175) that we can choose two hyperfuchsian functions, relative to a group $G$, such that every other function belonging to the same group is an algebraic function of the two chosen. Whether or not the two functions (15) are suitable for that purpose cannot be determined without a knowledge of the fundamental region for $G$. 
The several values of $w$ will be connected by the relation

$$
w_{m}=\theta^{(m-1)} w_{1}, \quad \theta=e^{\frac{2 \pi i}{\nu}} .
$$

Denote by $u$ any integral of the first kind, and by $u^{(m)}$ the value of $u$ taken over any path starting from a point in sheet $m$. Then

$$
u^{(m)}=\int \frac{R(z) d z}{w_{m}^{\eta}}=\int \frac{R(z) d z}{\theta^{(m-1)} w_{1}^{\eta}}=\theta^{\rho_{m}} \int \frac{R(z) d z}{w_{1}^{\eta}}=\theta^{\rho_{m}} u^{(1)},
$$

where $(m-1) \eta+\rho_{m} \equiv 0(\bmod \nu)$, with $0 \leqq \rho_{m}<\nu$.

By a simple circuit about a branch-point is meant a loop which starts and ends at $z_{0}$ in two different sheets which are successive in the order of the winding of the sheets for that branch-point. Denote by $\alpha^{(m)}, \beta^{(m)}, \ldots$, the values of $u^{(m)}$ along simple circuits about the branch-points $a, b, \ldots$, respectively. The values of $\alpha^{(m)}, \beta^{(m)}, \ldots$, for the different values of $m$ are connected by the relation (17) as follows: $\alpha^{(m)}=\theta^{\sigma_{m} \alpha^{(1)}}, \beta^{(m)}=\theta^{\sigma_{m}^{\prime}} \beta^{(1)}, \ldots$, where $\sigma_{m}, \sigma_{m}^{\prime}$, etc., are defined similarly to $\rho_{m}$. Since any closed path on the surface can be resolved by continuous deformation into a set of simple circuits about the branch-points, it follows that the value of $u^{(m)}$ along any closed path may be expressed in the form

$$
c_{1}^{\prime} \alpha^{(1)}+c_{2}^{\prime} \beta^{(1)}+\cdots+c_{\mu}^{\prime} \lambda^{(1)}
$$

where $c_{e}^{\prime}$ is a sum of roots of unity. For the integrals along the several simple circuits about $a$ may each be expressed in the form $\pm \theta^{\sigma_{m}} \alpha^{(1)}$, depending on the direction in which the integral is taken; consequently the portion of the value of $u^{(m)}$ due to paths around $a$ is of the form $c_{1}^{\prime} \alpha^{(1)}$ where $c_{1}^{\prime}$ is a sum of roots of unity. Similarly for the other branch-points. In particular, take $u$ positively over a path around all the branch-points, and we obtain a relation of the form

$$
c_{1} \alpha^{(1)}+c_{2} \beta^{(1)}+\cdots+c_{\mu} \lambda^{(1)}=0
$$

since the given path may be deformed into a point path.

Assume an appropriate set of branch-lines and cross-cuts, and define $A_{j}, B_{j}$, as in $\S 1$. Since the moduli of periodicity of $u$ are the results of integrating $u$ along these cross-cuts of the surface, we have as the values of $A_{j}, B_{j}$

$$
\begin{aligned}
& A_{j}=c_{1 j} \alpha^{(1)}+c_{2 j} \beta^{(1)}+\cdots+c_{\mu j} \lambda^{(1)}, \\
& B_{j}=d_{1 j} \alpha^{(1)}+d_{2 j} \beta^{(1)}+\cdots+d_{\mu j} \lambda^{(1)}, \quad(j=1,2, \cdots, p),
\end{aligned}
$$

where $c_{i j}, d_{i j}$ are constants. Substitute the value of $\lambda^{(1)}$ from (18) in the equations (19) and we have $2 p$ equations expressing the moduli of periodicity $A_{j}, B_{j}$ in terms of $\mu-1$ quantities $\alpha^{(1)}, \beta^{(1)}, \ldots$ Not more than $\mu-2$ of the $2 p$ moduli $A_{j}, B_{j}(j=1,2, \cdots p)$ are linearly independent. To prove this, assume that $\mu-1$ of them are independent. Solve these $\mu-1$ linearly independent 
equations for each of the $\mu-1$ quantities $\alpha^{(1)}, \beta^{(1)}, \ldots$. Thus we find that each of the quantities $\alpha^{(1)}, \beta^{(1)}, \ldots$ is expressed as a linear function of $\mu-1$ constants $A_{j}, B_{j}$. In particular, then,

$$
\alpha^{(1)}=\text { const. }
$$

But since $\alpha^{(1)}$ is the value of $u^{(1)}$ along an open path on the Riemann surface, it is a function of the end points and is not constant. Consequently the assumption made is false, and we have the theorem: Not more than $\mu-2$ of the moduli of periodicity of an abelian integral of the first kind associated with a binomial Riemann surface having $\mu$ branch-points are linearly independent. ${ }^{*}$

\section{§5. The fundamental transformations for the group of the general binomial surface.}

In $\S 1$ certain fundamental transformations arising from the monodromy of the branch-points of a Riemann surface were determined, and from them the generating tranformations of the group were found. The method employed there will be generalized in the present section; by analyzing separate cases we are finally led to the general theorem. We assume the conventions and definitions of $\S 1$.

Consider the binomial Riemann surface

$$
w^{\nu}=\left(z-a_{1}\right)^{a}\left(z-a_{2}\right)^{a} \cdots\left(z-a_{m}\right)^{\alpha},
$$

where $m \alpha \equiv 0(\bmod \nu), 0<\alpha<\nu, \alpha$ and $\nu$ integers and relative primes. Let $z_{0}$ be any arbitrarily chosen point of the surface, and with it as center swing a radius vector counter-clockwise ; let the branch-points be numbered $1,2, \cdots, m$, in the order in which the radius vector meets them. Denote by $(k l)=(l k)$ the transformation produced on any set of variables, functionally connected with the branch points $a_{k}, a_{l}$ of the surface, due to the interchange of these branchpoints. In the following discussion the symbol stands for the interchange itself. The interchange of the points is to be made in the manner indicated on pp. 40, 41.

If $a_{i}, a_{k}, a_{l}$ are three branch-points such that $i<k<l$, then $(i l)$ is equivalent to either $t_{1}=(i k)^{-1}(k l)(i k)$ or $t_{2}=(k l)(i k)(k l)^{-1}$. For in $t_{1},(i k)^{-1}$ means the interchange of $a_{i}$ and $a_{k}$ clockwise, then $(k l)$ the interchange of $a_{\imath}$ and $a_{k}\left(\equiv a_{i}\right)$ counter-clockwise, thus putting $a_{i}$ in the place of $a_{l}$, then $(i k)$ the interchange of $a_{k}\left(\equiv a_{l}\right)$ and $a_{i}\left(\equiv a_{k}\right)$ putting $a_{l}$ in place of $a_{i}$ and the path of $a_{k}$ may be deformed into a point path without passing over a branchpoint: the paths of $a_{i}$ and $a_{k}$ are between $z_{0}$ and $a_{l}$. A similar analysis verifies $t_{2}$. An analogous procedure applies if four or more branch-points are permuted to obtain an interchange. In particular, if $k<l$, we may express $(k l)$ in terms

\footnotetext{
* See Fricke-KLein, Automorphe Functionen, II, p. 135, where the essential statement of this theorem for $\mu=4$ is credited to J. Whelstein.
} 
of successive interchanges of branch-points, and obtain

$$
\begin{aligned}
(k l)=(k, k+1)^{-1}(k+1, k+2)^{-1} & \\
& \cdots(l-2, l-1)^{-1}(l-1, l)(l-1, l-2) \cdots(k+1, k) .
\end{aligned}
$$

It follows that the transformations due to every simple interchange can be expressed in terms of $m-1$ transformations $(i, i+1), i=1,2, \cdots, m-1$.

We will next discuss all other admissible types of motions of the branchpoints and the corresponding transformations of the variables. Let $a_{k}$ perform a complete loop about $a_{l}$; the resultant transformation is $(k l)^{2}$, for the complete loop is the succession of two interchanges of the points $a_{k}$ and $a_{l}$. In such a case the path of neither point can be deformed to a point-path. Next assume that $a_{k}$ and $a_{l}(k<l)$ are interchanged so that a branch-point $a_{i}$ is enclosed in the total path of the other points. The resultant transformation is

$$
(k i)(i l)(k i)=(i l)(k i)(i l),
$$

for in either case the path of $a_{i}$ is a point-path, that of $a_{l}$ is between $z_{0}$ and $a_{i}$ while $a_{i}$ is between $z_{0}$ and that of $a_{k}$. If $a_{j}$ is situated similarly to $a_{i}$ then the resulting transformation is

$$
(k i)(i j)(j l)(i j)(i k)=(l j)(j i)(i k)(j i)(j l) .
$$

In case $a_{i}$ lies between $z_{0}$ and the paths of $a_{k}, a_{l}$, the corresponding transformation is

$$
(k i)(i l)(i k)^{-1}=(i l)^{-1}(i k)(i l),
$$

and similarly for more points situated like $a_{i}$.

By an argument similar to that on p. 41 we may resolve any motion of any number of branch-points into a sequence of motions of the types discussed above, and may consequently express the resultant transformations in terms of $m-1$ successive interchanges designated above.

Next consider the binomial Riemann surface

$$
w^{\nu}=\left(z-a_{1}\right)^{\alpha} \cdots\left(z-a_{m}\right)^{\alpha}\left(z-b_{1}\right)^{\beta} \cdots\left(z-b_{n}\right)^{\beta},
$$

where $0<\alpha \neq \beta<\nu, m \alpha+n \beta \equiv 0(\bmod \nu), \alpha, \beta$, and $\nu$ are integers, and either $\alpha$ or $\beta$ is relatively prime to $\nu$. In a manner similar to that above we may select two sets of $m-1, n-1$ transformations in terms of which all the transformations due to all motions of the points $a$ among themselves, and to the points $b$ among themselves may be expressed. Denote by $T_{12}$ the transformation arising from the motion of some point $a$ around some point $b$ once. Evidently the points can be so chosen that the last point of the set $a$ moves around the first point of the set $b$. Then every transformation arising from proper motions of the points $a, b$ can be expressed in terms of the $m+n-1$ indicated transformations. To prove this it will evidently be sufficient to express the 
transformation arising from the motion of an arbitrary point $a$ around an arbitrary point $b$. Let $a_{i}$ and $b_{j}$ be the points. Interchange $a_{j}$ and $a_{m}, b_{j}$ and $b_{1}$; then use $T_{12}$, which puts $a_{m}$ around $b_{1}$; then interchange $a_{i}$ and $a_{m}, b_{j}$ and $b_{1}$, care being taken as to the direction of interchange. This completes the motions, and all paths except those of $a_{i}, b_{j}$ are point-paths, thus furnishing the required representation of the transformation. In general the number $m+n-1$ of fundamental transformations cannot be reduced. If, however, $n=1$, then $T_{12}=(m, m-1)^{-1} \cdots(12)^{-2} \cdots(m, m-1)^{-1}$, and the number of fundamental transformations is not greater than $m-1$.

In the case of several sets of branch-points $m_{1}, m_{2}, \ldots, m_{n}$ in number, which cannot be interchanged, a fundamental transformation will be required corresponding to the motion of some point of a set around a point of every other set. The corresponding transformations will be designated by $T_{k l}$, where $k$ and $l$ are different and refer to the above $n$ sets of branch-points. The number of these will be $\frac{1}{2} n(n-1)$, and this number will diminish by unity if some $m_{i}$ is unity. The number of transformations of each set is known from the above discussion to be $m_{l}-1$. To sum up, we have the following theorem:

The number of generators of the group arising from all possible motions of the branch-points of the binomial Riemann surface

where

$$
w^{\nu}=\prod_{m_{1}}\left(z-a_{i}\right)^{a_{1}} \prod_{m_{2}}\left(z-b_{i}\right)^{\alpha_{2}} \cdots \prod_{m_{n}}\left(z-l_{i}\right)^{a_{n}}
$$

$$
0<\alpha_{i} \neq \alpha_{j}<\nu, \quad \sum_{i=1}^{n} m_{i} \alpha_{i} \equiv 0 \quad(\bmod \nu),
$$

$\alpha_{i}$ and $\nu$ are integers, and some $\alpha_{i}$ is relatively prime to $\nu$, does not exceed

$$
\sum_{i=1}^{n} m_{i}-n+\frac{n(n-1)}{2}=\sum_{i=1}^{n} m_{i}+\frac{n(n-3)}{2} .
$$

If a detailed knowledge of an individual case is available, this number may be reduced. The group of $\S 1$ is an instance in point.

CoRnell University, Ithaca, N. Y. 